\title{
Characterization of Sputtered Shape Memory Alloy Ni-Ti Films by Cross- sectional TEM and SEM
}

R. M. S. Martins ${ }^{*, * *}$, A. Mücklich ${ }^{*}$, N. Schell ${ }^{* *}$, R. J. C. Silva ${ }^{* * *}$, K. K. Mahesh ${ }^{* * *}$ and F. M. Braz Fernandes $^{* * *}$

${ }^{*}$ FZD, P.O. Box 510119, 01314 Dresden, Germany

** GKSS, Max-Planck-Str. 1, 21502 Geesthacht, Germany

${ }^{* * *}$ CENIMAT/I3N, Campus da FCT/UNL, 2829-516 Monte de Caparica, Portugal

Ni-Ti Shape Memory Alloys (SMAs) have been attracting attention as smart materials because they can work as sensors and actuators at the same time. Miniaturization of mechanical devices is evolving toward sub-micron dimensions raising important questions in the properties of Ni-Ti films. In thin films it is essential to investigate the microstructure to understand the origin of the thickness limit. The design of functionally graded films has also been considered but for their successful development it is important to characterize the variations in crystalline structure [1].

We have explored the feasibility of a sputtering chamber designed to be mounted on the 6-circle goniometer of the ROBL beamline at the ESRF in order to follow by in situ XRD the evolution of the structure of the growing film. These experiments have shown the benefit of this technique to reveal intermediate "states" during the growth [2]. Here, we show complementary results obtained by X-TEM and SEM after deposition. X-TEM observations were carried out on a Philips CM300 microscope with $\mathrm{LaB}_{6}$ filament at $300 \mathrm{keV}$ and SEM observations on a Hitachi S-4800 with cold field emission cathode.

The morphology of the interface revealed the presence of $\mathrm{NiSi}_{2}$ with evidence that Ni diffuses preferentially along $<111>$ when analysing the depositions on $\mathrm{Si}(100)$ and $\mathrm{Si}(111)$ substrates (see figure 1). The electrical resistivity characterization of Ni-Ti graded films (deposited by controlled change of the ratio $\mathrm{Ti} / \mathrm{Ni}$ during deposition) has shown that the major part of the film shall be transformed to R-phase at RT [3]. The detection of the austenitic phase near the interface film/substrate at RT suggests that a minimum thickness is necessary to guarantee apparent phase transformation in the films presumably due to effects imposed by the interfacial diffusion layers.

The effect of a bias voltage on the growth of near equiatomic Ni-Ti films has been investigated by SEM. An increase of the substrate bias voltage from $-45 \mathrm{~V}$ to $-90 \mathrm{~V}$ led to the appearance of surface steps on the crystals (see figure 2 (a) and 2 (b)). A negative bias voltage causes a higher energetic ion bombardment of the growing surface, resulting in an enhanced surface-atom mobility but at sufficiently high voltage leads to defect formation. The study of the temperature dependence of the electrical resistivity has shown that the degree of ion bombardment alters the phase transformation behaviour of the films. The results are consistent with the assumption that spatial constraints introduced by the higher energetic bombardment $(-90 \mathrm{~V})$ in the microstructure of the film could generate a resistance force for lattice distortion and twinning. SEM has also been used to study the surface morphology of Ni-Ti films after the phase transformation to martensite. Figure 2 (c) shows a typical micrograph of a film exhibiting the martensitic phase and, thus, revealing a distinct morphology from that of austenite presented in figure 2 (a) (both films deposited with a substrate bias voltage of $-45 \mathrm{~V})$. 
Acknowledgements

This work was supported by the FCT/MCTES (Ph.D. scholarship, POCI 2010/FSE for R.M.S.M.), the DAAD (financial support under contract D/05/50641) and by the FZD.

\section{References}

[1] Y. Fu, H. Du, W. Huang, S. Zhang, M. Hu, Sens. Actuators A 112 (2004) 395.

[2] R.M.S. Martins, N. Schell, M. Beckers, K.K. Mahesh, R.J.C. Silva, F.M.B. Fernandes, Appl. Phys. A 84 (2006) 285.

[3] R.M.S. Martins, N. Schell, A. Mücklich, H. Reuther, M. Beckers, R.J.C. Silva, L. Pereira, F.M.B. Fernandes, Appl. Phys. A 91 (2008) 291.
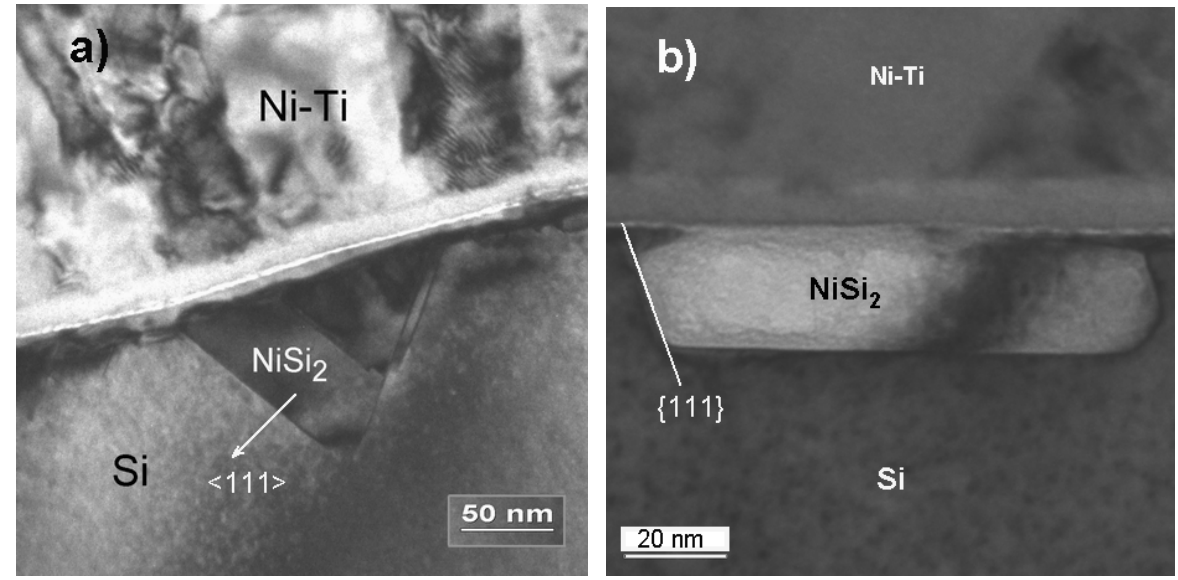

Figure 1. X-TEM micrographs of the interface region of Ni-Ti samples on Si recorded along the $\mathrm{Si}[110]$ zone axis. (a) Ni-Ti on $\mathrm{Si}(100)$. (b) Ni-Ti on $\mathrm{Si}(111)$. In micrograph (b) the $\mathrm{NiSi}_{2}$ compound show up as bright regions due to the particular alignment with the Si substrate. A white line was inserted to emphasize the orientation relationship between $\mathrm{NiSi}_{2}$ and the $\mathrm{Si}$ substrate since it shows an angle of approximately $70^{\circ}$ with the substrate surface (i.e., with the $\{111\}$ planes of Si).

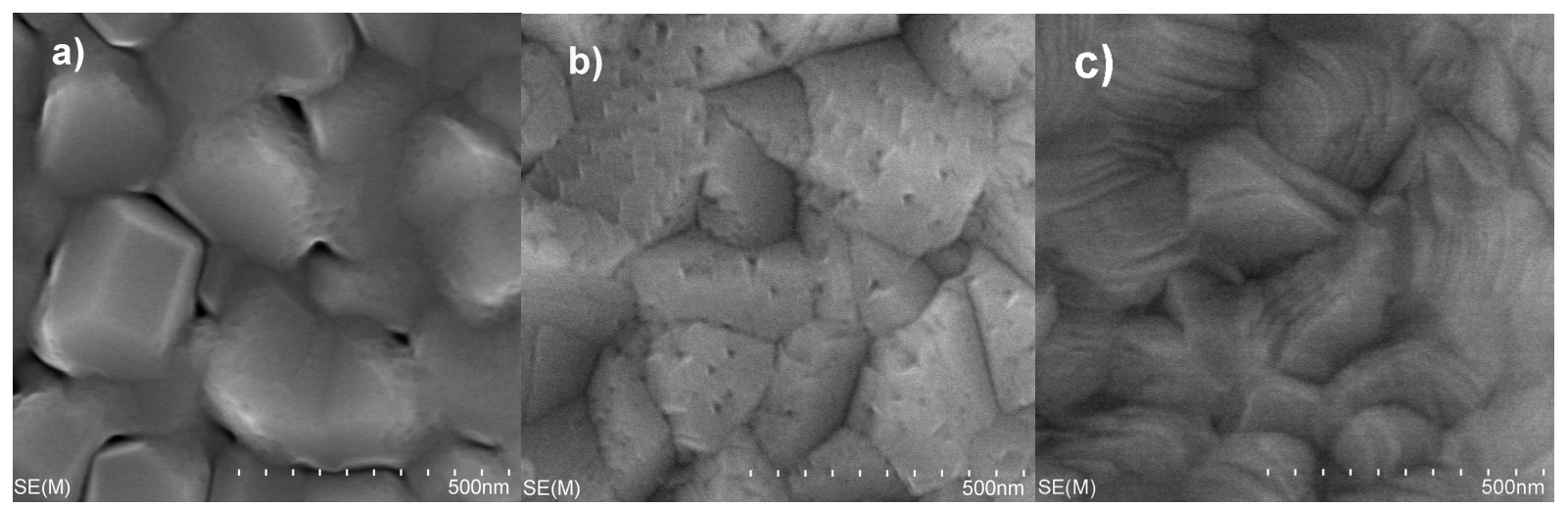

Figure 2. SEM micrographs showing the surface morphology of Ni-Ti films deposited on a TiN buffer layer. (a) Ni-Ti film deposited with a substrate bias voltage of $-45 \mathrm{~V}$ (at the austenitic state). (b) Ni-Ti film deposited with -90V (at the austenitic state). (c) Ni-Ti film deposited with -45V (at the martensitic state). 